\title{
A novel four-step system for screening angiogenesis inhibitors
}

\author{
QIN ZHOU $^{1 *}$, CUI-LING QI $^{1 *}$, YAN LI $^{2}$, XIAO-DONG HE ${ }^{1}$, JIANG-CHAO LI ${ }^{1}$, \\ QIAN-QIAN ZHANG ${ }^{1}$, LAN TIAN $^{1}$, MING ZHANG ${ }^{1}$, ZHE HAN $^{1}$, \\ HUIPING WANG ${ }^{1}$, XUESONG YANG ${ }^{2}$ and LI-JING WANG ${ }^{1}$
}

\author{
${ }^{1}$ Vascular Biology Research Institute, Guangdong Key Laboratory of Pharmaceutical Bioactive Substances, \\ Guangdong Pharmaceutical University, Guangzhou, Guangdong 510006; ${ }^{2}$ Division of Histology and Embryology, \\ Medical College, Jinan University, Guangzhou, Guangdong 510632, P.R. China
}

Received April 10, 2013; Accepted August 13, 2013

DOI: $10.3892 / \mathrm{mmr} .2013 .1704$

\begin{abstract}
Angiogenesis exhibits a significant effect on tumor progression. Inhibiting angiogenesis may provide significant advantages over currently available therapeutics for cancer therapies thus, the development of a system of screening angiogenesis is essential. In the present study, a novel available system of screening angiogenesis inhibitors by four steps was developed. The chorioallantoic membrane (CAM), yolk sac membrane and early chick embryo blood island assay were initially performed to obtain possible antitumor compounds. The MMTV-PyMT transgenic breast cancer mouse model was used for final screening and to confirm potential antitumor effects. Four angiogenesis inhibitors were isolated from 480 compounds, which were obtained from ICCB known bioactives library, by a combination of the CAM, yolk sac membrane and early chick embryo blood island assay. The MMTV-PyMT mouse was treated with one of four agents and it was demonstrated that the tumor volume was significantly inhibited. These results demonstrate that the four-step screening system is feasible.
\end{abstract}

Correspondence to: Dr Li-Jing Wang, Vascular Biology Research Institute, Guangdong Key Laboratory of Pharmaceutical Bioactive Substances Guangdong Pharmaceutical University, 280 Waihuandonglu, Guangzhou, Guangdong 510006, P.R. China

E-mail: wanglijing62@163.com

Dr Xuesong Yang, Division of Histology and Embryology, Medical College, Jinan University, 601 Huangpu Road West, Guangzhou, Guangdong 510632, P.R. China

E-mail: yangxuesong@126.com

*Contributed equally

Key words: chorioallantoic membrane, angiogenesis inhibitor screening system, yolk sac membrane, blood island model, MMTV-PyMT mouse

\section{Introduction}

Angiogenesis, the formation of novel microvasculature by capillary sprouting, occurs when hemangioblasts (the common pre-cursor cells of embryonic erythrocytes and endothelial cells) differentiate in the area vasculosa and the primary capillary plexus forms in situ (1). The significance of angiogenesis in tumor progression is well known. Vasoganglions supply blood and nutrition aiding solid tumor growth, and determining whether necrosis and apoptosis or invasion and metastasis of tumor cells occurs. The growth and metastasis of malignant cell populations also rely on the development of microvessels to grow and metastasize (2). There have been numerous studies concerning angiogenesis inhibitors. Sunitinib exhibited broad and potent antitumor activity by targeting the vascular endothelial growth factor receptor (3), with angiogenesis inhibitors, Marimastat and TNP-470, exhibiting antitumor activity in preclinical studies $(4,5)$. However, these inhibitors each have different limitations. There is an urgent requirement for a more efficient system for screening angiogenesis and angiogenesis inhibitors.

The allantois of the chick embryo appears at $\sim 3.5$ days of incubation (6). An extremely rich vascular network develops in chorioallantoic membrane (CAM) from day 4 to 14 (7). Thus, the chick embryo CAM may be used to study the angiogenic and angiogenic inhibitory activity of drugs and macromolecules (8). Macromolecules and compounds, such as a variety of growth factors (fibroblast growth factor-2, transforming growth factor- $\beta$ and tumor necrosis factor- $\alpha$ ) and protein kinase $c$ were demonstrated to induce CAM angiogenesis (9-11). CAM is used to investigate tumor angiogenesis and metastasis. However, as the chick's immune system is not completely developed at the early stages of chick embryo formation (12), the CAM assay has limitations including non-specific inflammatory reactions (13) and rearrangement of existing vessels that follows contraction of the membrane (14). These limitations influence the observations of vasoproliferative responses. Bouins and paraformaldehyde fluid solution are often used to repair the CAM for $\geq 3 \mathrm{~h}$ after the shell has separated from the membrane (15) as damage to the membrane and morphological changes in CAM occur easily during these operations. 
The capillary plexus in the yolk sac vascular tree develops into arteries and veins on days 3-5 of embryonic development (16). Consequently, the yolk sac membrane assay, which was essentially adapted from the CAM assay, was exploited. Polyamines and spermidine were observed to induce angiogenesis in the chick yolk sac membrane (17). The yolk sac membrane model was also utilized for studying the effect of radiation on vascular density in a rapidly growing tissue (18); however, there are a few studies concerning the chicken yolk sac membrane model for screening angiogenic and angiogenic inhibitors. Methylcellulose disk or drugs containing disk-shaped support are commonly used as drug containers and are placed on the yolk sac membrane (19). However, accurate results are not achieved when using this method as these containers cannot limit the area on the yolk sac membrane that is influenced by the drug.

In early vertebrate embryos, hematopoietic and endothelial lineages are derived from blood islands (aggregates of mesodermal cells) in the extra-embryonic yolk sac (20). Following the differentiation of blood island cells into blood and endothelial lineages, endothelial cells from individual blood islands anastomose to form the primary vascular plexus. The chick embryo remains a powerful model for studying developmental hematopoiesis and erythropoiesis (21). Although blood island formation is essential to analyze the early developmental stage of the embryonic blood vessels, to the best of our knowledge, the early embryonic blood island assay has not previously been utilized for anticancer drug screening.

FVB/N-Tg (MMTV-PyMT) 634Mul-transgenic mice, under the regulation of the mouse mammary tumor virus (MMTV) long terminal repeat, express the mouse polyomavirus middle-T antigen (PyMT). These transgenic animals consistently develop multifocal mammary tumors and exhibit a high incidence of pulmonary metastasis (22). In MMTV-PyMT mice, four distinctly identifiable stages of tumor progression from premalignant to malignant stages occur in a single primary tumor focus. Therefore, this model is a powerful tool for studying tumor progression (23).

In the present study, using the chick embryo CAM model, chick yolk sac membrane model, early chick embryo blood island assay and the MMTV-PyMT tumor model as a four-step screening system, potential angiostatic drugs were screened and the influence of these drugs on tumor development and tumor progression was investigated.

\section{Materials and methods}

Animals. Fertilized white leghorn chicken eggs were obtained from the Avian Farm of South China Agriculture University (Guangzhou, China), and were incubated under conditions of $50-60 \%$ humidity and $37.5^{\circ} \mathrm{C}$ when the shell had been cleaned with $1 \%$ geramine.

FVB/N-TgN (MMTV-PyMT) 634Mul-transgenic mice have been described previously (22) and were obtained from the Jackson Memorial Laboratory (Bar Harbou, MA, USA). All animals were weaned and the tails were clipped at 4 weeks of age. The mice were genotyped by PCR using a primer pair specific for the MMTV-PyMT transgene and distributed randomly into the treatment and control groups ( $\mathrm{G} 0$ treatment group, $\mathrm{N}=7$; DMSO control group, $\mathrm{N}=8$ ). The mice were housed and cared for in accordance with the 2011 Office of Animal Care and Use NIH guidelines.

Reagent. Four hundred and eighty compounds for the CAM assay were purchased from Enzo Life Science (Shenzhen, China; Lot \#3-P6574m) (Fig. 1). All 480 compounds were marked with a drug code (A0-A19; B0-B19; ... X0-X19) and dissolved in dimethylsulfoxide (DMSO; Amresco Inc., Solon, $\mathrm{OH}$, USA) to prepare a5 $\mathrm{mg} / \mathrm{ml}$ stock solution. The $\mathrm{G} 0$ and G2 drugs to be tested by the yolk sac membrane, blood island assay and MMTV-PyMT mice assays were purchased from Sigma-Aldrich (St. Louis, MO, USA). D1 was purchased from Enzo Life Science.

Chick embryo chorioallantoic membrane assay. The CAM assay was conducted as described previously, but with modifications (24). On the 9th day, the shell area beyond the air chamber of the egg was marked. Small windows $\left(10 \times 10 \mathrm{~mm}^{2}\right)$ were opened in the center of the marked area using a slow-speed dental molar which is used in dental surgery to smooth the teeth (STRONG90; Yijia Health Medical Devices Ltd., Gansu, China). The egg shells were then opened with sterile forceps. The sample solution [50 $\mu \mathrm{g} / \mathrm{ml}$ of experimental compounds and the negative control $0.3 \mu \mathrm{l}$ DMSO in $30 \mu \mathrm{l}$ phosphate-buffered saline ( $\mathrm{pH} \mathrm{7.4)],} \mathrm{were} \mathrm{then} \mathrm{placed} \mathrm{directly} \mathrm{onto} \mathrm{the} \mathrm{CAM}$ through the egg shell openings. The windows were sealed with a medical proof fabric medical proof fabric (Shanghai Medical Device Ltd., Shanghai, China; Lot \#080901) and the eggs were returned to the incubator at $37.8^{\circ} \mathrm{C}$ and $60 \%$ humidity. The egg shells were then cut carefully along the axis of the median line and the content was discarded after $48 \mathrm{~h}$. The CAM vasculature around the egg shell windows was observed and the treated area was defined as the area on the CAM where the compounds or control DMSO were attached. Digital images were captured at room temperature with a Canon camera (Tokyo, Japan). The images were analyzed and the number of every treatment condition (10 embryos per condition) in which the vessels had become less dense around the treated area was recorded as a positive number.

Chick embryo yolk sac membrane assay. On the 3rd day of incubation at $37.8^{\circ} \mathrm{C}$ and $60 \%$ humidity, chicken embryos were transferred to a sterilized culture dish. Eggs where the chicken embryo vessels faced upward were selected. Two silica gel circles with red and black marker, respectively, were placed in a symmetrical position of the yolk sac membrane of the chicken embryos. The culture dish was covered tightly prior to returning it to the incubator at $37.8^{\circ} \mathrm{C}$ and $60 \%$ humidity. A total of $30 \mu \mathrm{l} \mathrm{drug}(50 \mu \mathrm{g} / \mathrm{ml}$ in gelatin) was administered into the circles of the healthy embryos which had well-developed chicken embryos vessels $3 \mathrm{~h}$ later. Images of the vessels in the circles were captured by Image acquisition system OPTPRO 2007 (Guangpujia Technology Ltd., Beijing, China) at 0, 12 and $24 \mathrm{~h}$, respectively, following drug administration. The images were quantitatively analyzed by Image-Pro Plus 6.0 (Media Cyberneticss, Inc., Rockville MD, USA). The vessel growth rate in the DMSO-treated silica gel circle was treated as one. A t-test was conducted to show the difference between the vessel growth rate in the control and experimental groups. 


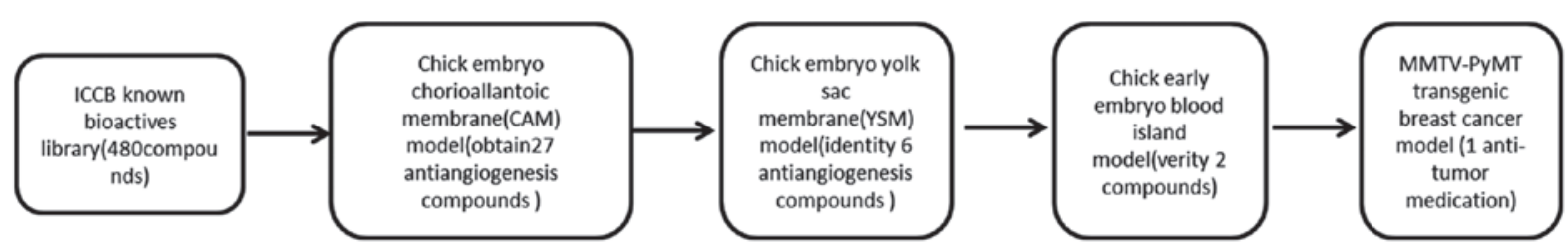

Figure 1. Schematic diagram of the four-step screening from angiogenesis inhibitors.

Early chick embryo blood island assay. Half of the chicken embryos were exposed to the medium containing drug at stages HH3-5 (25). The agar protein medium was separated equally into two containing test compound or control solvent. Graded concentrations $(0.025,0.05$ and $0.1 \mathrm{mg} / \mathrm{ml})$ of DMSO, G0 and D1 were mixed into different sides of the agar protein medium, then the early embryo was placed in the treated medium. Following $28 \mathrm{~h}$ of incubation at $37.8^{\circ} \mathrm{C}$ and $60 \%$ humidity and $4 \%$ paraformaldehyde fixing, a vascular endothelial (VE)-cadherin plasmid (obtained from the laboratory of C.J. Weijer, University of Dundee, Dundee, UK) was used for whole-mount in situ hybridization. The techniques used for in situ hybridization were essentially as described previously (26). The embryos were embedded with glutin-sucrose and cut into serial sections. The results were observed under an Olympus stereomicroscope (SZX16 Olympus, Tokyo, Japan).

MMTV-PyMT mice treatment. Glipizide or DMSO $(100 \mu \mathrm{g} / 20 \mathrm{~g}$ mouse weight) was injected intraperitoneally into the 9-week-old MMTV-PyMT mice every three days. Tumors were measured every four days and tumor volume was calculated as: Length $\mathrm{x}$ width ${ }^{2} \mathrm{x} 0.52$. After 28 days, the mice were sacrificed and the tumors were collected for weight analysis.

Immunohistochemistry. Tumor tissue sections were stained with goat polyclonal antibodies against CD31 (Santa Cruz Biotechnology, Inc., Santa Cruz, CA, USA; SC-1506) according to the manufacturer's instructions. Corresponding secondary antibody was purchased from Sigma-Aldrich and used according to the manufacturer's instructions. The blood vessel density was determined by quantifying the number of CD31-positive capillaries in 10 fields of vision from each section of different areas of the tumor (magnification, x200).

Statistical analysis. To examine the independent and normal distribution, the Student's t-test was used to determine whether if certain responses (immunohistochemical results, spontaneous tumor volumes and tissue weights) were influenced by drug treatment. $\mathrm{P}<0.05$ was considered to indicate a statistically significant difference.

\section{Results}

Twenty-seven potential angiogenesis inhibitors were obtained through high-throughput screening of 480 compounds utilizing the CAM assay. Ten embryos were used for each condition and the positive number in each group was recorded. All 480 compounds were marked with a drug code (A0-A19; B0-B19; ... X0-X19). Twenty-seven potential angiogenesis
Table I. High-throughput screening of 480 compounds by utilizing the CAM assay.

\begin{tabular}{ll}
\hline Positive no. & \multicolumn{1}{c}{ Compound code } \\
\hline 6 & A10, E10 \\
5 & C9, G0, G6, H4 \\
4 & B2, C5, D1, E7 \\
3 & B5, B6, B10, C6, D3, D4, D7, D8, E4, \\
& E11, G2, G4, H6, H8, H11, P1, P3 \\
\hline
\end{tabular}

Twenty-seven potential angiogenesis inhibitors were identified. CAM, chorioallantoic membrane. Bold, drug was used for further angiostatic activity screening.

inhibitors were isolated from the 480 compoundsobtained from the ICCB known bioactives library (Table I and Fig. 1).

Drug G0, G2 and D1 induce the inhibition of angiogenesis in the CAM assay. Twenty-seven potential angiogenesis inhibitors were obtained from 480 bioactive compounds by high-throughput screening based on the results of the CAM assay (Table I). In G0-treated CAM, the quantity and density of vessels decreased in the drug-treated area in five CAMS $(\mathrm{n}=10)$. The number of experimental CAMS treated with D1 and G2 which demonstrated angiogenic inhibition was 4 and 3 , respectively $(n=10)$. No vascular reaction was detectable in CAMS treated with DMSO alone (negative control) (Fig. 2). This showed that the application of G0, G2 and D1, but not DMSO suppressed the formation of neovasculature in chick embryo CAM.

G0, G2 and D1 induced the inhibition of angiogenesis of the embryo yolk sac membrane. The percentage of available embryos was $60 \%$ of the total when the chicken embryo contents were transferred to the sterilized culture dish. Thirty percent of the total quantity of the chicken embryos had well-developed vessels following $12 \mathrm{~h}$ of drug treatment. The yolk sac membrane from these embryos was observed, representative images were captured (Fig. 3B) and statistical analysis was conducted (Fig. 3C-E). The vessel growth rate following treatment with $\mathrm{G} 2, \mathrm{G} 0$ or D1 was notably decreased after a 12-h incubation with these drugs $(n=7, P=0.0295 ; n=9$, $\mathrm{P}=0.0013$ and $\mathrm{n}=7, \mathrm{P}=0.0301$, respectively), but not significantly decreased after $24 \mathrm{~h}$ incubation with $\mathrm{G} 2(\mathrm{n}=7, \mathrm{P}=0.1073)$.

$D 1$ and GO inhibited the development of the chicken embryo blood island in the early embryo model in vivo. Following incu- 
A

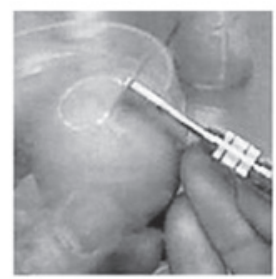

DMSO

B

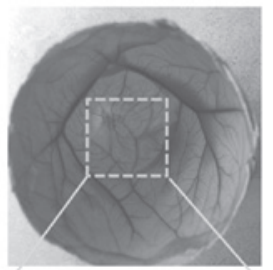

C

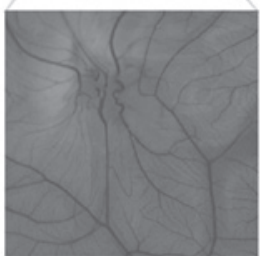

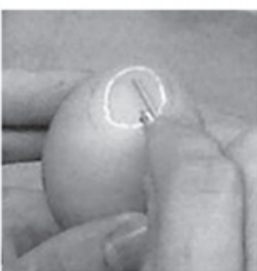

G2
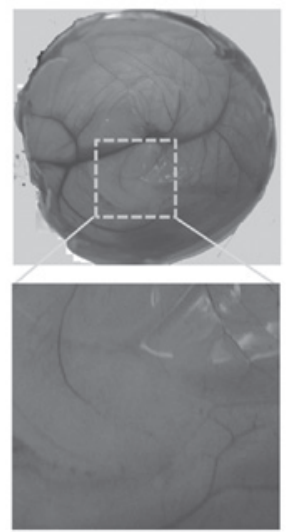

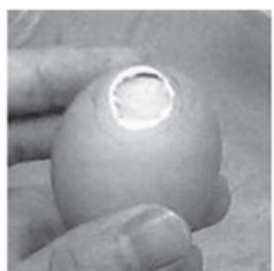

G0
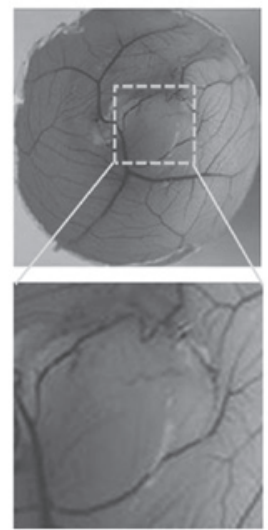

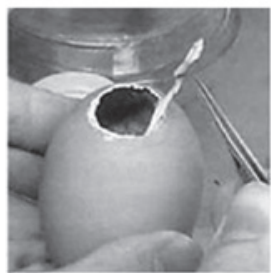

D1
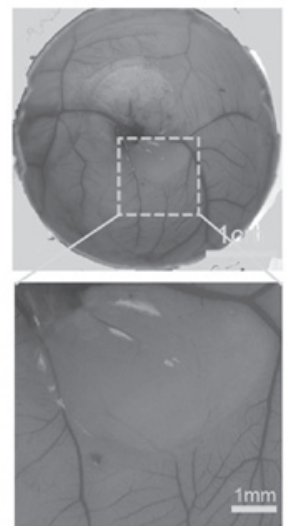

Figure 2. Compared with dimethylsulfoxide treatment, G2, G0 and D1 treatment inhibited the angiogenesis of chick embryo choriallantoic membrane (CAM) in the CAM assay. (A) CAM model protocol. (B) Images show the overall view of CAM $48 \mathrm{~h}$ following treatment. (C) Images are views of the area that was treated by the drugs.

A
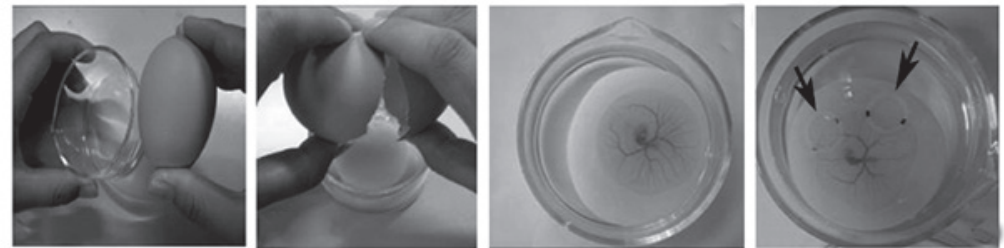

B
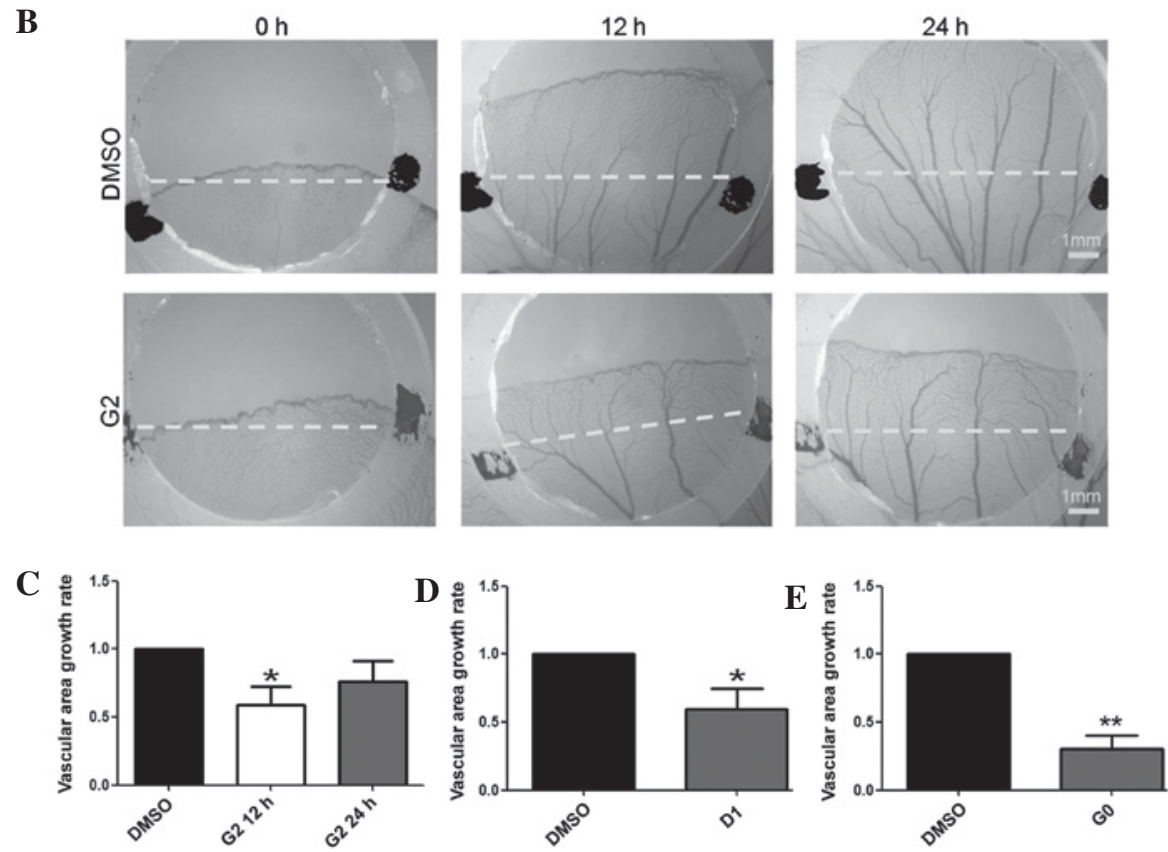

Figure 3. Compared with dimethylsulfoxide (DMSO) treatment, G2, G0 and D1 treatment inhibited the angiogenesis of the chicken embryo yolk sac membrane. (A) Yolk sac membrane model protocol. (B) Digital images of silica gel circles of vessels on chicken embryos yolk sac membrane captured by the Image acquisition system OPTPRO 2007 ( 0,12 and $24 \mathrm{~h}$ after treatment). The area covered by black marked silica gel circles was treated with DMSO and the red-marked area was treated with G2. (C) The vascular area growth rate was analyzed according to the image captured by the Stereo Microscope. Compared with DMSO treatment, G2 treatment inhibited angiogenesis of the yolk sac membrane after $12 \mathrm{~h}\left({ }^{*} \mathrm{P}=0.0295, \mathrm{n}=7\right)$, but not $24 \mathrm{~h}$ after treatment ( $\mathrm{P}=0.1073$, $\mathrm{n}=7$ ). (D) Compared with DMSO treatment, D1 treatment inhibited the angiogenesis of yolk sac membrane after $12 \mathrm{~h}$ treatment ( $\mathrm{P}=0.0301, \mathrm{n}=9)$. (E) After $12 \mathrm{~h}, \mathrm{G} 0$ treatment inhibited the angiogenesis of YSM compared with DMSO treatment $\left({ }^{* *} \mathrm{P}=0.0013, \mathrm{n}=7\right) .{ }^{*} \mathrm{P}<0.05$ and ${ }^{* * *} \mathrm{P}<0.001$. 
A

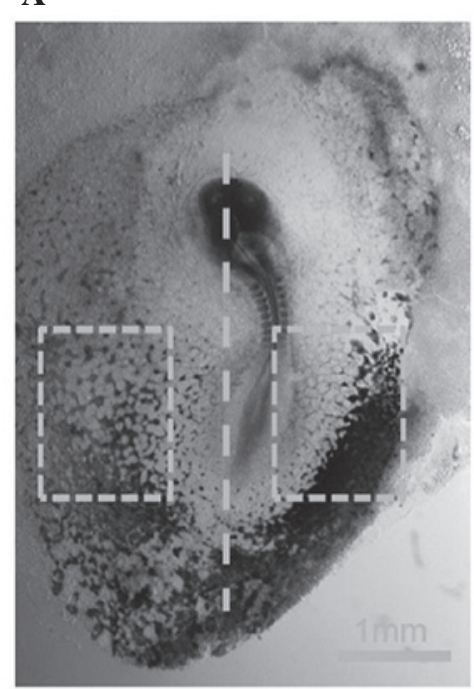

D

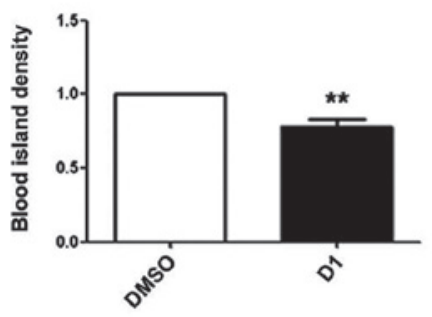

B

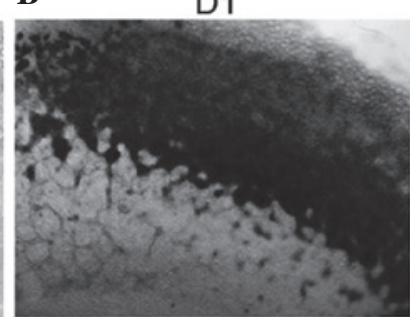

C DMSO

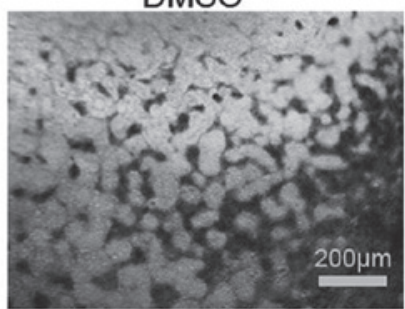

$\mathbf{E}$

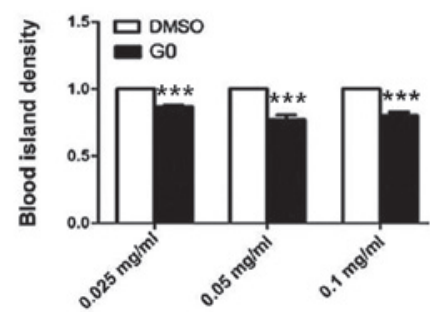

Figure 4. Compared with dimethylsulfoxide (DMSO) treatment, D1 and G0 treatment inhibited the development of the chicken embryo blood island. (A) Overall view of migration and the formation of the blood island in early chicken embryos. The image shows a chicken embryo section following vascular endothelial-cadherin in situ hybrization technique. (B and C) Blood islands following D1 and DMSO treatment. (D) The blood island density of the area treated with D1 $(0.05 \mathrm{mg} / \mathrm{ml})$ compared with that treated with DMSO. $(\mathrm{P}=0.0032, \mathrm{n}=8)$. (E) The blood island density of the area treated with three different concentrations of G0 $(0.025,0.05$ and $0.1 \mathrm{mg} / \mathrm{ml})$ compared with the area treated with DMSO $\left({ }^{* * *} \mathrm{P} \leq 0.0001, \mathrm{n}=10\right)$.

A

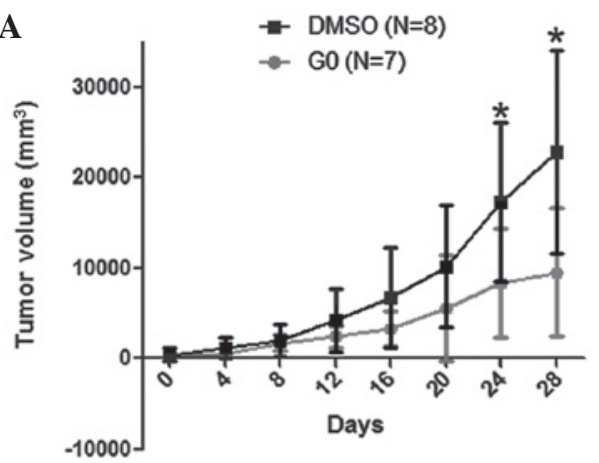

C

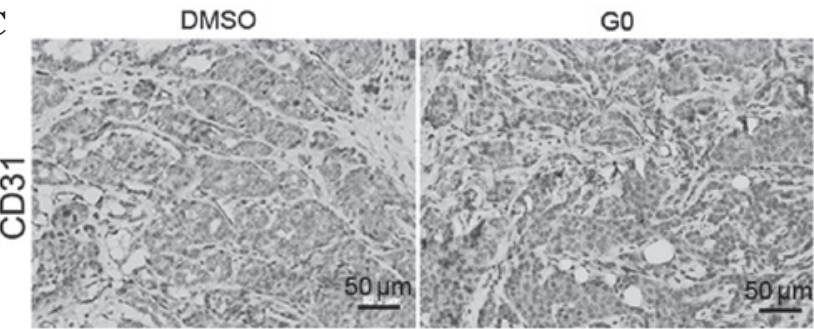

B

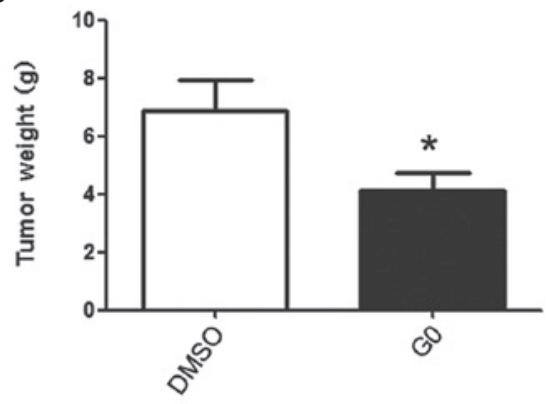

D

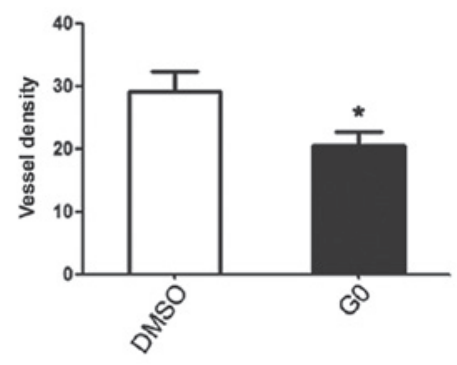

Figure 5. G0 attenuated breast cancer growth by inhibiting tumor angiogenesis. (A) The tumor volume of the MMTV-PyMT mice treated with dimethylsulfoxide (DMSO; $\mathrm{N}=8$ ) and $\mathrm{G} 0(\mathrm{~N}=7)$. (B) Weight of the tumors on day 28. (" $\mathrm{P}=0.0312$; DMSO group, $\mathrm{N}=14$; G0 group, $\mathrm{N}=15$ ). (C and $\mathrm{D}) \mathrm{CD} 31$ staining was used to visualize the functional vasculature in tumors from the mice treated with G0 and DMSO. Microvessel density was calculated by counting the number of vessels per field of each tumor in each mouse analyzed. $(\mathrm{P}=0.0357, \mathrm{n}=7){ }^{*} \mathrm{P}<0.05$, compared with DMSO treatment. 
bation, the blood islands were marked with VE-cadherin. The blood island density of the area treated with D1 $(0.05 \mathrm{mg} / \mathrm{ml}$ concentration) compared with that treated with DMSO was significantly decreased ( $\mathrm{P}=0.0032, \mathrm{n}=8$ ) (Fig. 4D). The blood island density of the area treated with three different concentrations of G0 $(0.025,0.05$ and $0.1 \mathrm{mg} / \mathrm{ml})$ was significantly decreased compared with the DMSO-treated area $(\mathrm{P} \leq 0.0001$, n=10; Fig. 4E).

G0 alleviates the growth of spontaneous breast cancer through the inhibitory function of tumor angiogenesis in MMTV-PyMT mice. The G0-treated group exhibited a notable decrease in tumor volume after 24 days treatment (Fig. 5A). All breast tumors from each mouse were harvested after 28 days of treatment and the tumor weight was measured. The tumor weight of the G0 group was observed to be decreased $(\mathrm{P}=0.0312$; DMSO group, $\mathrm{n}=14$; G0 group, $\mathrm{n}=15$; Fig. 5B). Immunohistochemistry was used to stain the breast tumor tissue with CD31 antibody to locate the vessels (Fig. 5C). The quantity of stained vessels in each unit area was counted. The vessel density of the G0 group was also significantly reduced compared with the DMSO control group $(\mathrm{P}=0.0357, \mathrm{n}=7$, Fig. 5D).

\section{Discussion}

In the CAM assay, compounds demonstrate an angiogenic function in the form of increased vessel density around the implant, with the vessels radially converging towards the center similar to the spokes of a wheel (27). However, when the quantity and density of vessels decreases and eventually disappears around the implant, an inhibitory function of the tested compound is shown (28). The instrument and device required for the CAM assay are easily obtainable. In our assay, the membrane was not detached from the shell or fixed as described in other studies. Since the embryo inflammation and experimental time were both reduced with this method, the procedure and acquisition of results were simple and br. An air chamber was also selected to add and locate the drugs for a reliable result. Using this method, to initially screen drugs and macromolecules with angiogenic and angiogenic inhibitory activity among compounds in the library is feasible and effective, and renders the high-throughput screening of 480 compounds possible.

The yolk sac membrane model is more sensitive to chemical compounds and uses the same organism as the CAM model, thus providing verification of the CAM results. In the present study, silica gel circles were used to limit the area influenced by drug treatment. Silica gel circles from the control and experimental groups were placed symmetrically on the same chicken embryo yolk sac membrane to avoid individual differences. In the current study, a morphometric evaluation, utilizing a computerized system, was undertaken to quantify the growth of blood vessels in the chicken yolk sac area vasculosa. This provides greater credibility to the test results.

Blood island formation is an important marker to assess the development of the early embryo blood vessels. The early embryo blood island assay demonstrates the inhibition of angiogenesis. At present, to the best of our knowledge, there are no studies regarding the function of the early embryo blood island assay in verifying angiogenesis inhibitors, thus, this study is the first to show it is valid.

Vascular angiogenesis is the most influential factor for determining blood supply and nutrition for solid tumor growth. Numerous angiogenesis inhibitory compounds and proteins are potential anticancer drugs. Thus, MMTV-PyMT transgenic mice, which spontaneously develops primary mammary tumor with four stages of tumor progression were used to investigate the antitumor effect of these compounds. The tumor volume, weight and microvessel density were analyzed. Through the CD31 staining to label the vessel of the tumor tissue, it is possible to determine whether the angiogenesis inhibitory effect of the compound influences tumor growth and progression.

Of 480 compounds, 27 inhibited angiogenesis in chick embryo CAM models. Using the chick embryo yolk sac membrane model and the blood island assay, three compounds were identified to exhibit significant angiogenic inhibition. One of these drugs was selected to verify the attenuation effect on the breast tumor growth in MMTV-PyMT transgenic mice by inhibiting angiogenesis.

In conclusion, a four-step system was created for screening angiogenesis inhibitors which combined the CAM, yolk sac membrane and early embryo island assays to identify potential angiogenic inhibitors and the spontaneous tumor genetically engineered mouse to investigate the potential antitumor effect of these drugs. Among 480 compounds from ICCB known bioactives library, three angiogenesis inhibitors were identified and one of these drugs was shown to exhibit antitumor activity by inhibiting tumor angiogenesis, which demonstrated that this system is feasible and effective. Thus, this study has demonstrated a four-step system for identifying compounds that inhibit the angiogenesis of tumors and may be developed as novel agents for the treatment of carcinoma.

\section{Acknowledgements}

The authors would like to thank Professor Jian-Guo Geng (University of Michigan) for providing the reagent and help with the discussion and Jie Ye and Lu Han for their technical assistance. This study was supported by the National Basic Research Program of China (project no. 973, grant no. 2010CB529702) and the Natural Science Foundation of China (grant no. 31271455 to Lijing Wang).

\section{References}

1. Risau W, Sariola H, Zerwes HG, et al: Vasculogenesis and angiogenesis in embryonic-stem-cell-derived embryoid bodies. Development 102: 471-478, 1988.

2. Weidner N, Folkman J, Pozza F, et al: Tumor angiogenesis: a new significant and independent prognostic indicator in early-stage breast carcinoma. J Natl Cancer Inst 84: 1875-1887, 1992.

3. Mendel DB, Laird AD, Xin X, et al: In vivo antitumor activity of SU11248, a novel tyrosine kinase inhibitor targeting vascular endothelial growth factor and platelet-derived growth factor receptors: determination of a pharmacokinetic/pharmacodynamic relationship. Clin Cancer Res 9: 327-337, 2003.

4. Toppmeyer DL, Gounder M, Much J, et al: A phase I and pharmacologic study of the combination of marimastat and paclitaxel in patients with advanced malignancy. Med Sci Monit 9: PI99-PI104, 2003.

5. Herbst RS: Targeted therapy using novel agents in the treatment of non-small-cell lung cancer. Clin Lung Cancer 3 (Suppl 1): S30-S38, 2002. 
6. Ribatti D, Nico B, Vacca A, Roncali L, Burri PH and Djonov V: Chorioallantoic membrane capillary bed: a useful target for studying angiogenesis and anti-angiogenesis in vivo. Anat Rec 264: 317-324, 2001

7. Ausprunk DH, Knighton DR and Folkman J: Differentiation of vascular endothelium in the chick chorioallantois: a structural and autoradiographic study. Dev Biol 38: 237-248, 1974.

8. Ribatti D, Vacca A, Roncali L and Dammacco F: The chick embryo chorioallantoic membrane as a model for in vivo research on angiogenesis. Int J Dev Biol 40: 1189-1197, 1996.

9. Wilting J, Christ B and Bokeloh M: A modified chorioallantoic membrane (CAM) assay for qualitative and quantitative study of growth factors. Studies on the effects of carriers, PBS, angiogenin, and bFGF. Anat Embryol (Berl) 183: 259-271, 1991.

10. Olivo M, Bhardwaj R, Schulze-Osthoff K, Sorg C, Jacob HJ and Flamme I: A comparative study on the effects of tumor necrosis factor-alpha (TNF-alpha), human angiogenic factor (h-AF) and basic fibroblast growth factor (bFGF) on the chorioallantoic membrane of the chick embryo. Anat Rec 234: 105-115, 1992.

11. Leibovich SJ, Polverini PJ, Shepard HM, Wiseman DM, Shively V and Nuseir N: Macrophage-induced angiogenesis is mediated by tumour necrosis factor-alpha. Nature 329: 630-632, 1987.

12. Leene W, Duyzings MJ and van Steeg C: Lymphoid stem cell identification in the developing thymus and bursa of Fabricius of the chick. Z Zellforsch Mikrosk Anat 136: 521-533, 1973.

13. Jakob W, Jentzsch KD, Mauersberger B and Heder G: The chick embryo choriallantoic membrane as a bioassay for angiogenesis factors: reactions induced by carrier materials. Exp Pathol (Jena) 15: 241-249, 1978.

14. Knighton DR, Fiegel VD and Phillips GD: The assay of angiogenesis. Prog Clin Biol Res 365: 291-299, 1991.

15. Ribatti D, Nico B, Vacca A and Presta M: The gelatin sponge-chorioallantoic membrane assay. Nat Protoc 1: 85-91, 2006.

16. le Noble F, Moyon D, Pardanaud L, et al: Flow regulates arterial-venous differentiation in the chick embryo yolk sac. Development 131: 361-375.

17. Takigawa M, Nishida Y, Suzuki F, Kishi J, Yamashita K and Hayakawa T: Induction of angiogenesis in chick yolk-sac membrane by polyamines and its inhibition by tissue inhibitors of metalloproteinases (TIMP and TIMP-2). Biochem Biophys Res Commun 171: 1264-1271, 1990.
18. Plasswilm L, Höper J, Cordes $\mathrm{N}$ and Tannapfel A: Investigation of microvessel density after irradiation. Radiat Res 151: 454-460, 1999.

19. Dias PF, Siqueira JM Jr, Maraschin M, Ferreira AG, Gagliardi AR and Ribeiro-do-Valle RM: A polysaccharide isolated from the brown seaweed Sargassum stenophyllum exerts antivasculogenic effects evidenced by modified morphogenesis. Microvasc Res 75: 34-44, 2008

20. Dieterlen-Lievre F: On the origin of haemopoietic stem cells in the avian embryo: an experimental approach. J Embryol Exp Morphol 33: 607-619, 1975.

21. Sheng G: Primitive and definitive erythropoiesis in the yolk sac: a bird's eye view. Int J Dev Biol 54: 1033-1043, 2010.

22. Guy CT, Cardiff RD and Muller WJ: Induction of mammary tumors by expression of polyomavirus middle $\mathrm{T}$ oncogene: a transgenic mouse model for metastatic disease. Mol Cell Biol 12: 954-961, 1992.

23. Lin EY, Jones JG, Li P, et al: Progression to malignancy in the polyoma middle $\mathrm{T}$ oncoprotein mouse breast cancer model provides a reliable model for human diseases. Am J Pathol 163: 2113-2126, 2003.

24. Chan T and Burggren W: Hypoxic incubation creates differential morphological effects during specific developmental critical windows in the embryo of the chicken (Gallus gallus). Respir Physiol Neurobiol 145: 251-263, 2005.

25. Hamburger V and Hamilton HL: A series of normal stages in the development of the chick embryo. 1951. Dev Dyn 195: 231-272, 1992.

26. Breier G, Albrecht U, Sterrer S and Risau W: Expression of vascular endothelial growth factor during embryonic angiogenesis and endothelial cell differentiation. Development 114 521-532, 1992.

27. Ribatti D, Urbinati C, Nico B, Rusnati M, Roncali L and Presta M Endogenous basic fibroblast growth factor is implicated in the vascularization of the chick embryo chorioallantoic membrane. Dev Biol 170: 39-49, 1995.

28. Iurlaro M, Vacca A, Minischetti M, et al: Antiangiogenesis by cyclosporine. Exp Hematol 26: 1215-1222, 1998. 